\title{
Editorial
}

\section{Modern Psychiatric Treatment: A Tribute to Thomas Detre, MD (1924-2011)}

\author{
Giovanni A. Fava \\ Affective Disorders Program, Department of Psychology, University of Bologna, Bologna, Italy; \\ Department of Psychiatry, State University of New York at Buffalo, Buffalo, N.Y., USA
}

Thomas Detre, one of the most influential psychiatrists of the past century, died after a long illness in 2011. He was born in Hungary in 1924. Unlike his family, he escaped death in the Holocaust. After the war, he moved to Italy and earned his medical degree in Rome in 1952. He then emigrated to the USA, where he became professor of psychiatry at Yale and, subsequently, at Pittsburgh. In this latter institution, as chairman of psychiatry he was able to build a leading department and as a vice chancellor for the health sciences he had a major influence in shaping the entire medical school.

I never had a chance to meet him in person, but I was very familiar with the book Modern Psychiatric Treatment he published with Henry Jarecki in 1971 [1]. Henry Jarecki was a psychiatrist at Yale; he then moved to a business career, to become a very successful entrepreneur and philanthropist. He currently lives between New York and the British Virgin Islands. I purchased the book in 1976, while an Italian medical student spending a summer elective in psychiatry in Dartmouth, and I have carried it with me since. It still has a special place in my personal library. It represents a unique blending of research evidence and clinical intuitions, one of the greatest exemplifications of the science of clinical judgment [2]. It derived from the magic encounter of two authors, much like the one that occurred in those years between John Lennon and Paul McCartney in music. The book was so much ahead of its time when it was published that, when I was consulting it for my clinical activities in the early 1980s, it did not really seem as if it had been written a decade earlier. When I browse through it today, I realize it is still ahead of its time, forty years later. How is it possible? Was it science fiction or has psychiatry failed to make substantial progress?

\section{The Book}

The book consisted of 685 pages on psychiatric assessment and treatment, including child psychiatry. In the first two chapters, the authors immediately explain how clinical judgment may be built and developed in psychiatric practice. The customary clinical taxonomy in psychiatry does not include patterns of symptoms, severity of illness, effects of comorbid conditions, timing of phenomena, rate of progression of illness, responses to previous treatments and other clinical distinctions that demarcate major prognostic and therapeutic differences among patients who otherwise seem to be deceptively similar since they share the same psychiatric diagnosis [2]. Let us consider some parts of the book.

\section{KARGER}

Fax +4161306 1234

E-Mail karger@karger.ch

www.karger.com
(C) 2012 S. Karger AG, Basel

0033-3190/13/0821-0001\$38.00/0

Accessible online at:

www.karger.com/pps
G.A. Fava, MD

Department of Psychology

Viale Berti Pichat 5

IT-40127 Bologna (Italy)

E-Mail giovanniandrea.fava@unibo.it 


\section{The Rollback Phenomenon}

In the section dedicated to 'Patterns of decompensation and remission' (pp. 52-54), Detre and Jarecki [1] provided a model for relating prodromal and residual symptomatology in psychiatric illness, defined as the rollback phenomenon; as the illness remits, it progressively recapitulates, though in reverse order, many of the stages and symptoms that were seen during the time it developed.

'For example, if an illness begins with occasional anxiety attacks that are superseded some weeks later by depressive symptoms which then become progressively more severe until, after several months, the patient develops total insomnia and confusion, the symptoms tend, as the condition improves, to remit in reverse order, the confusion and insomnia diminishing first, and the depressed mood next. After the depression lifts, the patient may again experience anxiety attacks for several weeks, until finally these symptoms, too, disappear [1, p. 54].'

According to the rollback model, there is also a temporal relationship between the time of development of a disorder and the duration of the phase of recovery: '... an illness that has arisen quickly is likely to recede quickly and, if it recurs, is likely to do so with the same rapidity that characterized previous episodes' [1, p. 95].

The rollback phenomenon was later substantiated in research on mood and anxiety disorders $[3,4]$. While developing the concept of sequential treatment in the early 1990s, I just had this in mind. If prodromal symptoms may have a pathophysiological role in affective disorders and some residual symptoms may progress to become prodromal symptoms of relapse [5], then reduction or disappearance of residual symptomatology may entail a more favorable long-term outcome of depression. A confirmation of this hypothesis was provided by a controlled therapeutic trial $[6,7]$, later confirmed by several independent investigations [8].

\section{Staging}

In the book, there is frequent reference to the concept of staging. For instance, in the progression of sleep disorders associated with psychiatric illness, Detre and Jarecki $[1, \mathrm{pp} .44-45]$ outlined two patterns of symptom development: type A disorders (exemplified by acute schizophrenic and manic episodes), where behavioral and sleep disturbances tend to arise, progress and remit relatively quickly, and type B illnesses (exemplified by depressive episodes), where both sleep disorders and mood symptoms develop and recede more slowly. However, the authors were careful in specifying that 'this dichotomization spells out only a trend ... The distinction indicates only that those features found in each group tend to cluster together, not that they must' $[1, \mathrm{p} .42]$.

Staging differs from the conventional diagnostic practice in that it does not only define the extent of progression of a disorder at a particular point in time but also where a person is currently located along the continuum of the course of illness [2]. When Robert Kellner and I developed staging methods for unipolar depression, mania, panic disorder and schizophrenia in 1993 [9], I had in mind many clinical examples from Modern Psychiatric Treatment [1].

\section{Diagnostic Approaches}

Clinical descriptions antedated DSM-III developments but were not that different. Let us look at how Detre and Jarecki [1] described the typical depressive episode: 'The typical depression is marked by symptoms of sadness, tearfulness, psychomotor retardation or agitation, anorexia, weight loss, diminished ability to concentrate, self-reproach, pessimism, diminished energy, libido, and interest, thoughts of death, suicidal ideas or attempts, insomnia and early morning awakening (EMA)' (pp. 162-163). Their diagnostic approach was not that of counting symptoms of a disorder and whether they cross an arbitrary cutoff, but to evaluate the extent to which a patient's clinical presentation matches a description of the disorder, the prototype diagnosis of psychiatric syndromes, which has been recently re-evaluated [10]. However, Detre and Jarecki were careful in pointing out that the typical description is in some ways an artifact and were very careful in outlining the full range of clinical presentations and their variability across age and multiple episodes. The same awareness of patient variability was applied to therapeutic responses (e.g. the likelihood of paradoxical reactions when using psychotropic drugs despite proper indications). The evaluations of overall severity, personal dysfunction and indications for treatment were guided by clinical judgment.

\section{Differential Diagnosis}

Attention was given to differentiations within a syndrome that may entail prognostic and therapeutic implications. For instance, in discussing delusional syndromes, the authors discriminated between type A delusions (that pervade all areas of the patient's thinking and are more frequent in younger persons and in those suffering from illness of relatively acute onset) and type B delusions (circumscribed, more frequent in older persons and in illnesses of slow onset) [1,pp. 205-206]. The tricks of clinical presentations were emphasized throughout the text. A complementary approach involved building unitary con- 
cepts from apparently scattered phenomena. Detre and Jarecki [1] dedicated one chapter to neurotic disorders, indicating that single diagnoses (e.g. obsessive compulsive and phobic neurosis) needed to be differentiated but with the awareness that what was shared by syndromes such as anxiety, panic, phobic disturbances and irritability might be as important as the differences between them and might constitute a prognostic indicator [11].

\section{What Happened to Clinical Judgment?}

While reading and consulting the book as a young psychiatrist, I always had the feeling that improvement in my performance was a function of upgrading my clinical judgment by trying to capture how senior colleagues and nurses came to certain conclusions and incorporating knowledge that could help me deal with complex and difficult clinical challenges. In this sense, Detre and Jarecki perfectly fit George Engel's description [12] of 'scientific physicians' (clinicians who fully apply the scientific method in their care of patients and in their understanding of the disease) as opposed to 'physician-scientists' (physicians whose primary commitment is to scientific research pertaining to medicine and who have little or no familiarity with the clinical process). Clinical practice is the source of fundamental scientific challenges for scientific physicians, whereas the application of basic (including pharmaceutical) research is the preferred focus of physician-scientists [12].

When reading Modern Psychiatric Treatment in the 1970s, one could foresee important developments in psychiatric assessment and treatment, an exciting progressive refinement of clinical skills with the support of research. However, the scenario was about to change in an opposite direction, leading to a progressive detachment from clinical observation and judgment [13].

The concept of evidence-based medicine, from its inception in the early 1990s, has achieved wide currency and enthusiastic endorsement in all areas of clinical medicine, including psychiatry [14]. The starting concern was reasonable; many patients were receiving ineffective treatments that were grounded in conventional practices, clinical intuition or physician idiosyncrasies rather than scientific evidence [15]. Feinstein and Horwitz [16] were among the first to warn about the dangers of excessive reliance on randomized controlled trials and meta-analyses. An issue which is neglected is the fact that, when transferred to clinical medicine from their origin in agricultural research, randomized trials were not intended to answer questions about the treatment of individual patients [16]. The results may show comparative efficacy of treatment for an average randomized patient but not for pertinent subgroups formed according to characteristics such as severity of symptoms, comorbidity and other clinical nuances.

\section{What Could Modern Psychiatric Treatment Be Today?}

In the early 1980s, discussing personality disorders, Robert Kellner [17] commented that classification systems have substantially increased agreement among diagnosticians because the conditions have been more clearly defined. He went on to write: 'However, agreement on a descriptive label does not necessarily mean agreement on a pathological entity (...). Sometimes the same underlying disorder can produce different clinical presentations (...). Conversely, different psychopathologies can lead to similar syndromes. For example the syndrome of persistent law-breaking, inability to learn from experience, and apparent disregard for group standards can stem in one person from the sociopathic traits of callousness, excessive egocentricity, and the absence of guilt; in another from impulsiveness and inadequate self-control; and in still another from emotional inadequacy (which prevents coping in a competitive society, and contributes to a guilt-ridden and fearful descent into crime)' [17, pp. 439-440]. Hence the difficulties in choosing an appropriate treatment strategy for the condition under examination. Similarly, biomarkers may be the result of different mechanisms, not necessarily reflecting a specific disease process [18]. Reliance on the genetic makeup of the individual is generally based on the assumption of a fixed condition over the individual's lifetime [19], whereas everything changes, both as a result of the natural course of a disease and its interactions with treatment [20]. If the application of biomarkers in medical practice should be guided by the concept of clinical utility (e.g. positive impact on patient outcome), how this can be determined is still an open question [18].

Unfortunately, current psychiatric treatment, also a result of massive doses of propaganda, is guided by the disease model, where identification of a DSM disorder should automatically lead to a specific 'cure'. Evidencebased medicine has then accurately minimized the disadvantages of each therapeutic ingredient, not only in terms of side effects, but also of contraindications. Its reductionistic approach centered on the average patient 
hides the wide fluctuations in treatment response that may occur. Horwitz et al. [21] developed a method of clinical inquiry within randomized controlled trials that can enhance the applicability of results to clinical decision making. Reanalyzing the Beta-Blocker Heart Attack Trial, they found that propranolol reduced the risk of dying for the 'average' patient who survived an acute myocardial infarction, whereas it was harmful in a subgroup of patients characterized by specific treatment histories. If we accept the possibility that a treatment which is helpful on average may be ineffective in some and even harmful for others, we may learn that a given therapy may not be of value for a particular class or subgroup of patients who are defined in terms of more detailed (compared to the randomized controlled trial eligibility criteria) specifications of clinical conditions. This may stimulate further research on alternative therapies that could potentially benefit the class of patients defined by the subgroup for whom otherwise effective therapy is offering no benefit or may even be causing harm [21]. Many examples may be provided in the field of psychopharmacology [22], as well as in psychotherapy research [23].

Not surprisingly, mainstream research in psychiatry has entailed little, if any, clinical progress, and endorsement of the principles of evidence-based medicine has disdainfully tagged as nonscientific any flexibility that clinical science requires. As it applies to other cultural and scientific fields, however, it is thinking out of the box that may generate a paradigm shift that is able to assure continuity with the efforts of Detre and Jarecki [1]. Fortunately, there have been developments in the science of clinical judgment in psychiatry that may make it possible to draw the scenario of a modern psychiatric treatment that departs from current reductionistic models.

\section{Diagnostic Formulations}

Feinstein [24] remarks that, when making a diagnosis, thoughtful clinicians seldom leap from a clinical manifestation to a diagnostic endpoint. The clinical reasoning goes through a series of 'transfer stations', where potential connections between presenting symptoms and the pathophysiological process are drawn. These stations are a pause for verification, or a change to another direction [24]. The use of diagnostic transfer stations has been suggested by the sequential treatment model [8], an intensive, two-stage approach that includes the use of a treatment (e.g. psychotherapy) after remission has been achieved with another (e.g. pharmacotherapy). The sequential model relies on repeated as- sessments (after each line of treatment has been completed) that may modify an initial diagnosis (e.g. preexisting anxiety disturbances may emerge after pharmacotherapy of a major depressive episode). It recognizes that for most patients, a single course of treatment is insufficient to yield adequate improvement and that different combined or sequential approaches may be necessary. Similarly, exclusive reliance on diagnostic criteria is suitable for relatively simple, well-circumscribed clinical pictures of new onset and in particular when it is important to determine whether the patient's disturbances are above or below a certain clinical threshold. However, when the clinical picture is complex, chronic and modified by courses of treatment, as in the majority of cases seen in psychiatric practice nowadays, different strategies should be endorsed.

\section{Assessment Strategies}

A method has been developed in psychiatry for organizing clinical data as variables in clinical reasoning. Emmelkamp et al. [25] introduced the concept of macroanalysis (a relationship between co-occurring syndromes and problems is established on the basis of where treatment should commence in the first place) in anxiety disorders. This method has been extended to mood disorders [26], psychosomatic assessment [27] and drug dependence [28]. Macroanalysis starts from the assumption that in most cases there are functional relationships with other more or less clearly defined problem areas and that the targets of treatment may vary during the course of disturbances [25-28]. Feinstein [29], introducing the concept of comorbidity, referred to any 'additional co-existing ailment' separate from the primary disease, even in the case that this secondary phenomenon does not qualify as a disease per se. Indeed, in clinical medicine, the many methods that are available for measuring comorbidity are not limited to disease entities [30]. As a result, macroanalysis expands the focus of clinical attention from the usual symptomatic suspects to psychological well-being, illness behavior, emotional inhibition, allostatic load, family relationships and work situation [2].

\section{Clinical Reasoning}

The link that is operated among these problem areas follows the routes of clinical reasoning and may involve: - establishing hierarchical relationships (e.g. the primary/secondary distinction based on chronological appearance of disturbances when depression occurs in the setting of anxiety); 
- building unitary concepts (conditions that are apparently comorbid could be part of the same clinical syndrome), as was subsumed under the rubric of neurosis, in its phenomenological [11] and psychodynamic [31] traditions;

- subtyping and differentiating within a diagnostic entity by incorporating elements of macroanalysis also within a diagnostic entity (e.g. anxious depression); the need to subtype major depressive disorder, since this category is too broad to yield meaningful treatment implications, has been recently underscored [32-34];

- staging of disturbances, whereby a disorder is characterized according to seriousness, extension and longitudinal development [9];

- determining the role of medical comorbidity (e.g. endocrine conditions that yield affective disturbances that are unlikely to respond to standard treatments) [35], and

- previous treatment history [20], including effects such as tolerance, sensitization and resistance [22], which constitute iatrogenic comorbidity.

\section{Microanalysis}

Clinical reasoning needs more information within a specific problem area and thus leads to microanalysis, a detailed analysis of specific symptoms (onset and course of the complaints, circumstances that worsen symptoms and consequences) [25]. For instance, when anxiety characterizes the clinical picture, it is necessary to know under which circumstances the anxiety becomes manifest, what the patient does when he/she becomes anxious, whether avoidant behavior occurs and what are the longterm consequences of the avoidance behavior. Microanalysis also consists of dimensional measurements, such as observer or self-rating scales for assessing anxiety and fears. Microanalysis is consequential and secondary to macroanalysis and leads to overcoming the assumption that there is a common assessment strategy for all clinical encounters (i.e. the obsolete concept of a psychometric battery). The use of biological markers in the setting of microanalysis may also be more clinically rewarding than its ideological application to ill-defined entities. Tomba and Bech [36] have specifically addressed the relationship between rating scales and macroanalysis, providing the clinimetric criteria to operate the choice of instruments. In clinimetrics, differential emphasis can be placed on symptoms, unlike in psychometrics, where all items are weighed the same [2]. In 1976, for instance, Bielski and Friedel [37] identified the predictors of a positive response to antidepressant drugs (insidious onset, upper socioeconomic class, anorexia, weight loss, middle and late insomnia and psychomotor disturbance). The presence, characteristics and intensity of specific symptoms may determine a redefinition of hierarchical relationships of problem areas and use of prototype diagnosis more than diagnostic criteria.

\section{Treatment Planning}

The planning of treatment thus requires determination of the symptomatic target of the first-line approach (e.g. pharmacotherapy) and tentative identification of other areas of concern to be addressed by subsequent treatment (e.g. psychotherapy). For instance, certain psychotherapeutic strategies can be deferred to a residual stage of depression, when state-dependent learning has been improved by the use of antidepressant drugs [8]. The hierarchical organization that is chosen may depend on a variety of factors (e.g. urgency, availability of treatment tools) that also include the patient's preferences and priorities. Macroanalysis is not only a tool for the therapist but can also be used to inform the patient about the relationship between different problem areas and motivate the patient to change. Such attention provides the background for maximizing the nonspecific ingredients of treatment, which are at least as important as those that are regarded as specific [2]. The concept of a shared decision is gaining increasing attention in clinical medicine [38] but is still seldom practiced in psychiatry [39].

\section{Treatment Selection}

Choice of a treatment ingredient (e.g. an antidepressant drug) is thus dictated by consideration of its advantages and disadvantages, which are determined by evaluation of the specific clinical situation and not by abstract consideration of 'diseases' and 'cures' [26]. The model is realistic instead of idealistic as in evidence-based medicine. The ill-defined concept of treatment resistance is based on the untested assumption that treatment was right in the first place and failure to respond is entirely shifted onto (and implicitly blamed upon) the patient's characteristics. Treatment resistance thus calls for switching and augmentation, instead of reconsideration of the process of treatment selection. The naïvete of this approach is reminiscent of early research on the placebo effect and its quest for identification of the 'placebo reactor', before landmark innovative studies identified the importance of the settings and patient-doctor interaction and demonstrated that the same subject may display a placebo response in one experimental condition and not in another [40-44]. 


\section{The Treatment Unit}

This model of psychiatric treatment requires that the psychiatrist does not operate in isolation, but in close cooperation with an internist and a few psychotherapists, who may provide treatment after the initial evaluation of the psychiatrist according to a sequential therapeutic plan [45]. Such a team may provide broader services to medical populations, potentially encompassing patients who lack an organic explanation for their disturbances [27]. The role of psychiatrists in the traditional public mental health clinics has been hampered by a perceived restriction of the psychiatrist's role to prescribing and signing forms, limiting opportunities to engage in the kind of integrated care that attracted many physicians to this specialty [45].

\section{A Leading Role for Psychiatry as a Medical Discipline?}

The concepts of macro- and microanalysis do not pertain only to clinical psychology and psychiatry but have been used with broader socioeconomic connotations. In particular, Charles Wright Mills argued that micro and macro levels of analysis can be linked together by the sociological imagination, i.e. the combination of private problems with public issues [46]. Individuals can only understand their experiences fully if they locate themselves within their period of history. It is the clinical imagination, as portrayed by Modern Psychiatric Treatment [1], that may entail a solution to the current impasse of psychiatric research and practice. The notion of psychiatric disease is not in line with the changed spectrum of health and the complex interplay of biological and psychosocial factors [2]. Evidencebased medicine may lead to undertreatment, overtreatment or mistreatment and is not geared to the complexity of clinical situations [13].

In the 1950s, the sociologist Harvey L. Smith defined the psychiatrist as the marginal man of the medical profession [47]. Psychiatry has been struggling against this marginality, but current emphasis on the role of biomarkers that should compensate the clinical inadequacies of psychiatrists are likely to reinforce this process further. Actually, in their clinical practice, psychiatrists use sophisticated forms of clinical judgment, master techniques of interviewing and history taking and are geared to integrating biological, psychological and social issues. However, these skills are minimized by current research strategies. Yet, fascinating vistas for clinical judgment are opening up. They should be welcome to all those who are disillusioned with the meager practical results of decades of mainstream psychiatric research and should become the preferred channel of funding and attention. Psychiatry could then lead other medical disciplines to an overdue reappraisal of evidence-based medicine and to a renewed interest in the key characteristics of clinical science in its explicit attention to humanness, where observation (outer-viewing), introspection (inner-viewing) and dialogue (inter-viewing) are the basic methodological triad for clinical assessment and for making patient data scientific [48].

\section{Disclosure Statement}

The author has no conflict of interest to declare.

\section{References}

1 Detre TP, Jarecki HG: Modern Psychiatric Treatment. Philadelphia, Lippincott, 1971.

-2 Fava GA, Rafanelli C, Tomba E: The clinical process in psychiatry: a clinimetric approach. J Clin Psychiatry 2012;73:177-184.

$\checkmark 3$ Fava GA: Subclinical symptoms in mood disorders: pathophysiological and therapeutic implications. Psychol Med 1999;29:47-61.

4 Fava GA, Mangelli L: Subclinical symptoms in panic disorder. Psychother Psychosom 1999;68:281-289.

5 Fava GA, Kellner R: Prodromal symptoms in affective disorders. Am J Psychiatry 1991; 148:823-830.
6 Fava GA, Grandi S, Zielezny M, Canestrari $\mathrm{R}$, Morphy MA: Cognitive behavioral treatment of residual symptoms in primary major depressive disorder. Am J Psychiatry 1994; 151:1295-1299.

-7 Fava GA, Grandi S, Zielezny M, Rafanelli C, Canestrari R: Four year outcome for cognitive behavioral treatment of residual symptoms in major depression. Am J Psychiatry 1996;153:945-947.

-8 Fava GA, Tomba E: New modalities of assessment and treatment planning in depression. CNS Drugs 2010;24:453-465.
-9 Fava GA, Kellner R: Staging: a neglected dimension in psychiatric classification. Acta Psychiatr Scand 1993;87:225-230.

10 Westen D: Prototype diagnosis of psychiatric syndromes. World Psychiatry 2012;11: $16-21$.

11 Tyrer P, Seivewright N, Ferguson B, Tyrer J: The general neurotic syndrome. Acta Psychiatr Scand 1992;85:201-206.

12 Engel GL: Physician-scientists and scientific physicians. Resolving the humanism-science dichotomy. Am J Med 1987;82:107-111.

13 Fava GA: Clinical judgment in psychiatry: requiem or reveille? Nord J Psychiatry 2012, E-pub ahead of print. 
14 Whitley R, Rousseau C, carpenter-Song E, Kirmayer LJ: Evidence-based medicine: opportunities and challenges in a diverse society. Can J Psychiatry 2011;56:514-522.

$\checkmark 15$ Sackett DL, Rosenberg WMC, Gray JAM, Haynes RB, Richardson WS: Evidence based medicine: what it is and what it isn't. BMJ 1996;312:71-72.

16 Feinstein AR, Horwitz RI: Problems in the 'evidence' of 'evidence-based medicine'. Am J Med 1997;103:529-535.

17 Kellner R: Personality disorders; in Greist JH, Jefferson JW, Spitzer RL (eds): Treatment of Mental Disorders. New York, Oxford University Press, 1982, pp 429-449.

18 Woodcock J: Assessing the clinical utility of diagnostics used in drug therapy. Clin Pharmacol Ther 2010;88:765-773.

19 De Leon J: Evidence-based medicine versus personalized medicine. Are they enemies? J Clin Psychopharmacol 2012;32:143-164.

20 Tomba E: Nowhere patients. Psychother Psychosom 2012;81:69-72.

-21 Horwitz RI, Singer BH, Makuch RW, Viscoli CM: Can treatment that is helpful on average be harmful to some patients? J Clin Epidemiol 1996;49:395-400.

-22 Fava GA, Offidani E: The mechanisms of tolerance in antidepressant action. Progr Neuropsychopharmacol Biol Psychiatry 2011;35: 1593-1602.

23 Cella M, Chalder T, White PD: Does the heterogeneity of chronic fatigue syndrome moderate the response to cognitive behavior therapy? An exploratory study. Psychother Psychosom 2011;80:353-358.

24 Feinstein AR: An analysis of diagnostic reasoning. I. The domains and disorders of clinical macrobiology. Yale J Biol Med 1973;46: 212-232.

25 Emmelkamp PMG, Bouman TK, Scholing A: Anxiety Disorders. Chichester, Wiley, 1993.

-26 Tomba E, Fava GA: Treatment selection in depression: the role of clinical judgment. Psychiatr Clin North Am 2012;35:87-98.
27 Fava GA, Sonino N, Wise TN (eds): The Psychosomatic Assessment. Karger, Basel, 2012.

28 Cosci F, Fava GA: New clinical strategies of assessment of comorbidity associated with substance use disorders. Clin Psychol Rev 2011;31:418-427.

29 Feinstein AR: The pre-therapeutic classification of comorbidity in chronic disease. J Chronic Dis 1970;23:455-468.

30 deGroot V, Beckerman H, Lankhorst GJ, Bouter LM: How to measure comorbidity: a critical review of available methods. J Clin Epidemiol 2003;56:221-225.

31 Taylor GJ: Affects, trauma, and mechanisms of symptom formation. A tribute to John C. Nemiah, MD (1918-2009). Psychother Psychosom 2010;79:339-349.

-32 Lichtenberg P, Belmarker RH: Subtyping major depressive disorder. Psychother Psychosom 2010;79:131-135.

33 Bech P: The struggle for subtypes in primary and secondary depression and their modespecific treatment or healing. Psychother Psychosom 2010;79:331-338.

34 Guidi J, Fava GA, Picardi A, Porcelli P, Bellomo, Grandi S, Grassi L, Pasquini P, Quartesan R, Rafanelli C, Rigatelli M, Sonino N: Subtyping depression in the medically ill by cluster analysis. J Affect Disord 2011;132: 383-388.

-35 Fava GA, Sonino N: Depression associated with medical illness. CNS Drugs 1996;5:175189.

-36 Tomba E, Bech P: Clinimetrics and clinical psychometrics: macro- and micro-analysis. Psychother Psychosom 2012;81:333-343.

37 Bielski RJ, Friedel RO: Prediction of tricyclic antidepressant response. A critical review. Arch Gen Psychiatry 1976;33:1479-1489.
38 Joosten EAG, De Fuentes-Merillas L, de Weert GH, Sensky T, van der Staak CPF, de Jong CAJ: Systematic review of the effects of shared decision-making on patient satisfaction, treatment adherence and health status. Psychother Psychosom 2008;77:219-226.

39 Joosten EAG, de Jong CAJ, de Weert-van Oene GH, Sensky T, van der Staak CPF: Shared decision making reduces drug use and psychiatry severity in substance-dependent patients. Psychother Psychosom 2009; 78:245-253.

40 Rickels K: Psychopharmacologic agents. J Nerv Ment Dis 1963;136:540-549.

41 Fava GA: The intellectual crisis of psychiatric research. Psychother Psychosom 2006;75: 202-208.

42 Gliedman CH, Nash EH, Huber SD, Stone AR, Frank JD: Reduction of symptoms by pharmacologically inert substances and by short-term psychotherapy. AMA Arch Neurol Psychiatry 1958;79:345-351.

43 Downing RW, Rickels K: Nonspecific factors and their interaction with psychological treatment in pharmacotherapy; in Lipton MA, Di Mascio A, Killam KF (eds): Psychopharmacology: A Generation of Progress. New York, Raven, 1978, pp 1419-1427.

44 Uhlenhuth EN, Rickels K, Fisher S, Park LC, Lipman RS, Mock J: Drug, doctor's verbal attitude and clinic setting in the symptomatic response to pharmacotherapy. Psychopharmacologia 1966;9:392-418.

45 Fava GA, Park SK, Dubovsky SL: The mental health clinic: a new model. World Psychiatry 2008;7:177-181.

46 Wright Mills C: The Sociological Imagination. Fortieth Anniversary Edition. Oxford, Oxford University Press, 2000.

$\checkmark 47$ Smith HL: Psychiatry in medicine: intra- or inter-professional relationships? Am J Sociol 1957;63:285-289.

48 Engel GL: From biomedical to biopsychosocial. Psychother Psychosom 1997;66:57-62. 